\title{
Preoperative inflammatory markers of NLR and PLR as an indicators of poor prognosis in resectable HCC
}

\author{
Dong Wang ${ }^{1}$, Ning Bai ${ }^{2}$, Xi Hu ${ }^{1}$, Xi Wu OuYang ${ }^{1}$, Lei Yao ${ }^{1}$, YiMing Tao ${ }^{\text {Corresp., } 1}$, ZhiMing Wang ${ }^{\text {Corresp. } 1}$ \\ 1 Department of General Surgery, Xiangya Hospital, Central South University, Hunan, China \\ 2 Department of Emergency, Xiangya Hospital, Central South University, Hunan, China \\ Corresponding Authors: YiMing Tao, ZhiMing Wang \\ Email address: yimingtao@csu.edu.cn, zhimingwang@csu.edu.cn
}

Background: Many recent studies have demonstrated the predominant role chronic inflammation plays in cancer cell propagation, angiogenesis and immunosuppression. Cancer-related inflammation (CRI) has been shown to correlate with poor cancer prognosis. Our study aimed to evaluate the prognostic value of the neutrophil-to lymphocyte ratio (NLR) and platelet-to-lymphocyte ratio (PLR) in patients with hepatocellularcarcinoma (HCC) who have undergone liver resection.

Methods: Between 2012 and 2015, 239 patients with HCC who had undergone liver resection at XiangYa Hospital Central South University were included in this study. The values of simple inflammatory markers including the NLR and PLR, used in predicting the long-term outcomes of these patients, were evaluated using Kaplan-Meier curves and Cox regression models.

Results: The cutoff values of the NLR and PLR were 2.92 and 128.1, respectively. In multivariate Cox regression analysis, high NLR ( $\geq 2.92)$ and high PLR $(\geq 128.1)$ were independent risk factors predicting poorer outcomes in patients with HCC. However, high NLR and high PLR were prognostic factors in tumor size and tumor number.

Conclusions: In this study, we identified that high NLR ( $\geq 2.92)$ and high PLR $(\geq 128.1)$ are useful prognostic factors in predicting outcomes in patients with HCC who underwent liver resection. 


\section{Preoperative inflammatory markers of NLR and PLR as an indicators of poor}

\section{Corresponding Author:}

8 YiMing Tao,

9 Changsha, Hunan, China

ZhiMing Wang, Changsha, Hunan, China

\section{Abstract} liver resection.

\section{prognosis in resectable $\mathrm{HCC}$}

\author{
Dong Wang ${ }^{1}$, Ning $\mathrm{Bai}^{2}$, Xi Hu${ }^{1}$,Xi Wu OuYang ${ }^{1}$, Lei Yao ${ }^{1}$, YiMing Tao ${ }^{1 \dagger}$, ZhiMing Wang ${ }^{1 \dagger}$ \\ ${ }^{1}$ Department of General Surgery, Xiangya Hospital, Central South University, Hunan, China. \\ ${ }^{2}$ Department of Emergency, Xiangya Hospital, Central South University, Hunan, China.
} address: yimingtao@csu.edu.cn.

Department of Hepatobiliary Surgery, Xiangya Hospital, Central South University, Email address: zhimingwang@,csu.edu.cn.

Background: Many recent studies have demonstrated the predominant role chronic inflammation plays in cancer cell propagation, angiogenesis and immunosuppression. Cancer-related inflammation (CRI) has been shown to correlate with poor cancer prognosis. Our study aimed to evaluate the prognostic value of the neutrophil-to lymphocyte ratio (NLR) and platelet-tolymphocyte ratio (PLR) in patients with hepatocellular carcinoma (HCC) who have undergone

Methods: Between 2012 and 2015, 239 patients with HCC who had undergone liver resection at XiangYa Hospital Central South University were included in this study. The values of simple inflammatory markers including the NLR and PLR, used in predicting the long-term outcomes of these patients, were evaluated using Kaplan-Meier curves and Cox regression models. Results: The cutoff values of the NLR and PLR were 2.92 and 128.1, respectively. In multivariate Cox regression analysis, high NLR $(\geqslant 2.92)$ and high PLR $(\geqslant 128.1)$ were independent risk 
30 factors predicting poorer outcomes in patients with HCC. However, high NLR and high PLR 31 were prognostic factors in tumor size and tumor number.

32 Conclusions: In this study, we identified that high NLR $(\geq 2.92)$ and high PLR $(\geq 128.1)$ are

33 useful prognostic factors in predicting outcomes in patients with HCC who underwent liver 34 resection. 


\section{Introduction}

36 Hepatocellular carcinoma (HCC) is the most common type of cancer and the third leading cause

37 of cancer-related death worldwide [1]. Hepatitis infection plays a leading role in $\mathrm{HCC}$

38 occurrence and progression [2]. Owing to a high occurrence of hepatitis B virus (HBV) and

39 aflatoxin infection, China alone accounts for approximately half of all HCC cases, making HCC

40 a major medical burden in our country. Hepatectomy and liver transplantation are considered as

41 curative treatments for HCC patients [3, 4] and despite improved diagnosis and advances in

42 surgical techniques, the clinical prognosis of HCC is still poor [5].

44 Recently, many studies have demonstrated that chronic inflammation plays a predominant role in cancer cell propagation, angiogenesis and immunosuppression [6]. Cancer-related inflammation (CRI) has been shown to correlate with poor cancer prognosis [7, 8]. Inflammation caused by EB

47 virus infection is related to nasopharyngeal cancer, hepatitis virus infection leads to HCC, and

48 Helicobacter pylori infection leads to gastric cancer. CRI helps cancer cells to acquire malignant biological behaviors, including proliferation, infiltration, angiogenesis, and metastasis. The nuclear factor $\kappa b(\mathrm{NF}-\kappa \mathrm{B})$ [9] and transcription activator 3 (STAT3) [10] pathways are well known in CRI. Chemokines, including TNF [11], CXCL8 [12], and IL-6 [13], also play an important role in the pathophysiological process of tumor formation. CRI parameters, including

53 C-reactive protein (CRP) [6], platelet-to-lymphocyte ratio (PLR) [14], and neutrophil-

54 lymphocyte ratio (NLR) [15], are widely used in cancer patients to guide treatment and predict prognosis. These biomarkers are more readily available and non-invasive.

57 However, the ability of the NLR and PLR to predict the prognosis of patients with HCC after liver resection is under debate. Our study was designed to combine the preoperative underwent curative resection. 
61 Materials \& Methods

62 Study population

63 Our study included 239 patients with HCC who underwent liver resection between 2012 and 642015 at XiangYa Hospital, Central South University, China. HCC was confirmed using

65 66 postoperative pathology. Patients with any one of the following items were excluded from this study: (1) had undergone splenectomy; (2) recurrence of HCC; (3) ruptured HCC; (4) infections during the perioperative period; (5) other autoimmune diseases; (6) preoperative antitumor treatments; and (7) preoperative application of interferon, interleukin or other similar drugs. This study was approved by the ethics committee of XiangYa Hospital Central South University (No. 201709984) and with the patients' informed consent.

Follow-up and definitions

Blood routine, liver function, serum alpha-fetoprotein (AFP), and hepatitis B surface antigen (HBsAg) were tested in all patients. Abdomen ultrasonography, computed tomography (CT) or magnetic resonance imaging (MRI), and chest radiography were performed for all patients. NLR measured neutrophil count to lymphocyte count, and PLR measured platelet count to lymphocyte count. Recurrence was diagnosed using imaging (CT or MRI) and AFP. An AFP level >20 $\mathrm{ng} / \mathrm{mL}$ was defined as being high [16]. The cutoff values of NLR and PLR were determined using receiver operating characteristic curves (ROC) according to the overall survival of patients. The seventh edition of the American Joint Committee on Cancer tumor-node metastasis (TNM) staging system and Barcelona Clinic Liver Cancer were applied to rank the HCC stage.

\section{Statistical analysis}

Statistical analyses were performed using Prism software (GraphPad Prism Software, La Jolla, CA) and SPSS 21.0 (SPSS Company, Chicago, IL) for Windows. Quantitative values were analyzed using $t$ tests. Categorical variables were compared using the chi-square test or Fisher's exact test. Recurrence-free survival (RFS) and overall survival (OS) were evaluated using the Kaplan-Meier method and the log-rank test. Prognostic factors of RFS and OS were analyzed using univariate and multivariate analyses [17]. $P<0.05$ was considered statistically significant. 


\section{Results}

92 Assessment of the cut-off value of NLR, PLR and LMR

93 According to the ROC curve, the ideal cutoff values for preoperative NLR and PLR were 2.92

94 and 128.1, respectively. The ROC areas under the curve for the NLR and PLR were 0.63 (95\%

$95 \mathrm{CI}$ for the area between 0.56 to 0.71$)$ and 0.67 (95\% CI for the area between 0.55 to 0.72 ),

96 respectively. The cutoff values of the NLR and PLR presented correspond to sensitivity values of

$9751 \%$ and $81 \%$, and specificity values of $78 \%$ and $42 \%$, respectively (Figure 1).

The relationship of clinical and pathologic characteristics with preoperative NLR and PLR

101 A total of 239 patients met the enrollment conditions, including 200 (83.68\%) males and 39

$102(16.32 \%)$ females, and were enrolled in the present study. As presented in Table 1, the mean age

103 was $(50.14 \pm 11.98)$ years. The mean tumor size was $(5.88 \pm 3.59) \mathrm{cm}$ and $56(23.43 \%)$ patients

104 had multiple tumors. A high preoperative AFP was observed in 155 (64.85\%) patients. HBV

105 surface antigen was positive in $202(84.5 \%)$ patients, $71(29.7 \%)$ patients had tumor

106 encapsulation, and 174 (72.8\%) patients had liver cirrhosis that was confirmed by pathology.

As shown in Table 1, the relationships between preoperative NLR and PLR, and between clinical and pathologic characteristics were investigated. The high-NLR group included 104 (43.51\%) patients (NLR $>2.92)$ and 135 (56.49\%) patients were identified as being in the low-NLR

111 (NLR $\leq 2.92$ ) group. Eighty-seven (36.4\%) patients were identified as being in the high-PLR

112 group (PLR $>128.1)$, and 152 (63.6\%) patients were identified as being in the low-PLR group

$113(\mathrm{PLR} \leq 128.1)$.

114

115 Preoperative NLR level and PLR level were closely correlated with tumor size, TNM stage and 116 BCLC stage $(P<0.05)$. The PLR also correlated with age, platelet count, prothrombin time $(\mathrm{PT})$, 117 AFP, and satellite nodules $(P<0.05)$. No obvious correlations with gender, HBsAg, hospital stay, 118 liver cirrhosis, serum albumin, total bilirubin (TBil), glutamic-pyruvic transaminase (ALT), or 119 glutamic-oxaloacetic transaminase (AST) were observed $(P>0.05)$.

120

121 The correlation between NLR, PLR and postoperative RFS and OS in patients with HCC 122 who underwent liver resection 
123 Kaplan-Meier survival analysis showed that the NLR $>2.92$ group was associated with a shorter

124 recurrence-free survival (RFS) (Figure 2A) and overall survival (OS) (Figure 2C). The patients

125 with HCC in the PLR>128.1 group were also associated with a shorter RFS (Figure 2B) and OS

126 (Figure 2D).

127

128 From the univariate analysis in Table 2, we found that tumor size (HR 1.30, 95\% CI 1.08-1.56),

129 NLR (HR 2.85, 95\% CI 1.63-4.93), PLR (HR 1.013, 95\% CI 1.00-1.02), BCLC stage (HR 3.005, $13095 \%$ CI 1.39-6.50), and satellite nodules (HR 4.27, 95\% CI 2.55-7.14) correlated with RFS in

131 patients with HCC who underwent liver resection $(P<0.05)$. The platelet count (HR 1.01, 95\% CI

132 1.00-1.01), AST (HR 1.02, 95\% CI 1.00-1.03), tumor size (HR 1.42, 95\% CI 1.23-1.63), NLR

133 (HR 1.48, 95\% CI 1.15-1.88), PLR (HR 1.007, 95\% CI 1.001-1.013), TNM stage (HR 19.42,

134 95\% CI 2.61-144.3), BCLC stage (HR 2.43, 95\% CI 0.99-5.98), satellite nodules (HR 4.42, 95\%

135 CI 2.66-7.33), and tumor number (HR 2.78, 95\% CI 1.18-6.54) correlated with OS $(P<0.05)$.

136 Gender, HBsAg, hospital stay, liver cirrhosis, serum albumin, total bilirubin (TBil), ALT and so

137 on had no statistically significant association with RFS or OS (P>0.05).

138

139 In the multivariate analysis, we found that NLR (HR 1.16, 95\% CI 1.06-1.26) and PLR (HR

$1401.004,95 \%$ CI 1.001-1.006) were independent risk factors for RFS in patients with HCC. NLR

141 (HR 1.14, 95\% CI 1.04-1.25) and PLR (HR 1.004, 95\% CI 1.001-1.007) were independent risk

142 factors for OS in patients with HCC.

143

144 Combined NLR and PLR to analyze RFS and OS in patients with HCC who underwent

145 hepatectomy

146 In the previous results, we found that high NLR and high PLR are independent risk factors for

147 RFS and OS after hepatectomy in patients with HCC. We combined NLR with PLR to

148 investigate whether the prediction of RFS and OS was more accurate. We defined NLR $\leq 2.92$ as

$149 \mathrm{NLR}_{\text {-low }}, \mathrm{NLR}>2.92$ as $\mathrm{NLR}_{\text {-high }}, \mathrm{PLR} \leq 128.1$ as $\mathrm{PLR}_{\text {-low }}$, and PLR>128.1 as $\mathrm{PLR}_{\text {-high. We found }}$

150 that patients with simultaneously high NLR and PLR had the worst RFS (median 12 months) and

151 OS (median 18 months), while patients with simultaneously low NLR and PLR had the best RFS

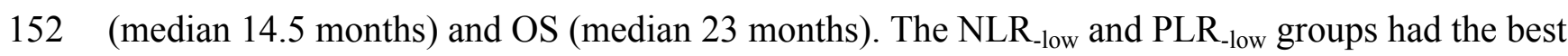

153 outcome and their RFS and OS were superior to other groups. The worst group was the NLR -high $_{\text {. }}$ 


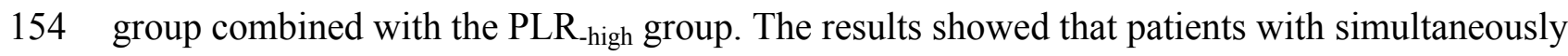

155 high NLR and high PLR were more prone to metastasis and had the worst OS (Figure 3).

156

\section{The relationship between NLR, PLR, tumor size, and satellite nodules}

158 Using multivariate analysis, we found that tumor size was an independent risk factor for poor

159 prognosis in patients with HCC who underwent liver resection. To see if there is any correlation, 160 we analyzed the relationship between NLR, PLR and tumor size. We divided the tumors into 161 three groups by size: $\leq 3 \mathrm{~cm}$ group, between $3-10 \mathrm{~cm}$ group, and $\geq 10 \mathrm{~cm}$ group. We found the 162 NLR and PLR were higher in groups with larger tumor size $(P<0.05)$ (Figure 4).

163 The mean NLRs in the tumor $\leq 3 \mathrm{~cm}$ group, $3-10 \mathrm{~cm}$ group and $\geq 10 \mathrm{~cm}$ group were $(2.32 \pm 0.15)$, $164(3.23 \pm 0.17)$, and (4.03 \pm 0.38$)$, respectively (Figure 4A); the mean PLRs were (90.21 \pm 6.44$)$,

165 (128.5 \pm 5.4$),(157 \pm 13.41)$, respectively (Figure 4B). We hypothesized that with high

166 neutrophil and platelet counts, cancer cells can release various chemokines and promote tumor

167 growth. At the same time, the number of lymphocytes decreased, and tumor cells escaped from

168 the immune surveillance, as the immune system could not activate its normal anti-tumor effect.

169 As a result, the HCC tumor growth progresses, increasing the tumor size.

170

171 We further analyzed the relationship between NLR, PLR and BCLC stage. We found that the 172 advantaged BCLC stage had higher NLR and PLR values. The mean NLR values of BCLC 0, A, $173 \mathrm{~B}$, and $\mathrm{C}$ stages were $(1.70 \pm 0.14),(2.93 \pm 0.13),(3.05 \pm 0.26)$, and (4.82 \pm 0.65$)$ (Figure 5A), 174 respectively. The mean PLR values of BCLC0, A, B, and C stages were (81.93 \pm 10.68$),(122.1$ $175 \pm 5.61),(122.5 \pm 8.91)$, and (149.2 \pm 16.13$)$, respectively (Figure 5B). 


\section{Discussion}

179 Many researchers have demonstrated that inflammation contributes to the pathogenesis and

180 progression of cancer [18]. The presence of systemic inflammation is associated with poor

181 survival in many types of tumors, and anti-inflammatory agents have been associated with cancer

182 prevention and treatment [19]. Inflammation can promote cancer development through multiple

183 mechanisms, including gene mutation, cancer cell proliferation and angiogenesis [7]. NLR and

184 PLR have been shown to have the ability to predict the prognosis in various cancers, including

185 HCC [20], esophageal carcinoma [21], renal carcinoma [22], and lung cancer [23]. Lu et al [20]

186 have studied the NLR in early and intermediate stage HCC, and in our research, we found the

187 NLR and PLR can predict the prognosis of patients with HCC who underwent liver research. The

188 stages included were early, intermediate and advanced stage $\mathrm{HCC}$, all showing similar results.

189

190 In solid tumors, inflammation often appears before the tissue malignant transformation. The

191 occurrence and development of systemic immune responses provide an appropriate

192 microenvironment for cancer metastasis and recurrence. In China, most patients with HCC have

193 hepatitis infection, the inflammatory status playing an important role in promoting the

194 development of HCC. The NLR and PLR, sensitive indexes of the body's inflammation system,

195 can reflect the inflammatory state and predict the prognosis of the tumor.

196

197 Neutrophil can strengthen the biological behavior of the tumor, causing it to grow and

198 metastasize. Higher neutrophil levels can upregulate the expression of growth factors, such as the

199 types of chemokines, which play an important role in tumor development and progression.

200 Platelets play a leading role in tumor progression. Platelets can secrete inflammatory factors,

201 including TGF- $\beta$ and VEGF, which can accelerate the differentiation and proliferation of tumor

202 cells. Moreover, platelets release platelet derived factors, such as platelet reactive protein, etc.,

203 which play an important role in tumor adhesion, and angiogenesis to (1) prepare the

204 microenvironment for tumor metastasis by secreting angiogenic factor and growth factor; and (2)

205 shield the cancer cell so the platelets can adhere to the tumor. Platelets can protect cancer cells

206 from the mechanical force of blood flow, and can also provide a shield for cancer cells that

207 allows them to escape immune surveillance.

208 
209 Many studies have confirmed that lymphocytes are the most important cells in tumor killing.

210 When there is a relative or absolute reduction of lymphocytes, the antitumor effect is also

211 decreased. PD-1 and CTLA-4 inhibitors are the most important immunity drugs [24, 25]. They

212 can reduce tumor cell and T lymphocyte cell interaction by inhibiting the cancer cell surface

213 expression of PD-1 and CTAL-4. The patient's decreased immunity, specifically the abnormality

214 of the tumor immune microenvironment, leads to the failure of the lymphocyte immune

215 response, and the cancer cells escape from immune surveillance. When immune tolerance or

216 immune escape occurs, tumors are more likely to progress or metastasize. In our study, we found

217 that patients with higher NLR and PLR had worse RFS and OS prognosis. On one hand, the

218 increase of neutrophil and platelet counts promotes tumorigenesis; on the other hand, the

219 decrease in the number of lymphocytes leads to the patient's immunity decline, leading to tumor

220 progression.

221

222 In our study, we found that tumor size correlated with the NLR and PLR; the larger the tumor

223 size, the higher the NLR and PLR. We hypothesize that (1) as neutrophil and platelet counts

224 increase, they secrete many kinds of growth factors and inflammatory factors, which promote the

225 growth of tumor cells and stromal cells and impact the tumor microenvironment and promote

226 tumor growth; and (2) larger tumor size means higher tumor burden. The number of lymphocytes

227 obviously decreased, and the effect of tumor cells killing is also weakened, thereby promoting

228 the development of the tumor. Tumor size is one of the prognostic predictors for patients with

229 HCC, but tumor size was more difficult to measure than the NLR and PLR. Additionally, tumor

230 size cannot provide an accurate prediction of HCC, because the NLR and PLR can reflect

231 whether patients with HCC are associated with cirrhosis and hypersplenism. If the tumor is

232 small, but liver cirrhosis and hypersplenism are obvious, the prognosis of patients with HCC will

233 be poor.

234

235

236 We also found that the BCLC stage correlated with high NLR and high PLR. The advanced

237 BCLC stages had higher PLR and NLR. Multiple tumors and/or vascular invasion in patients

238 with HCC may lead to a stronger inflammatory response and weaker immune response. Higher

239 neutrophil and platelet counts mean a stronger inflammatory response. Lower lymphocyte counts

240 mean the immune response is decreased, and cancer cells are more likely to metastasize. 
242 This study has some limitations. First, the number of patients in our study is small and the

243 patients were retrospectively studied in a single center. Therefore, we could not avoid selection

244 bias when collecting information on patients with HCC. Second, the NLR and PLR were

245 assessed by single measurements at the time of admission for the initial diagnosis.

246

247 Conclusion

248 In conclusion, our study showed that NLR and PLR are useful prognostic factors in predicting 249 outcomes in patients with HCC who underwent live resection. This finding can assist in guiding 250 the clinical management of patients with HCC.

251

252 Acknowledgements

253 We gratefully acknowledge all the authors' works for this paper and all the patients in our study. 254

255

\section{Reference}

256 [1] Ferlay J, Soerjomataram I, Dikshit R, Eser S, Mathers C, Rebelo M, Parkin DM, Forman D and Bray F.

[6] Chaturvedi AK, Caporaso NE, Katki HA, Wong HL, Chatterjee N, Pine SR, Chanock SJ, Goedert JJ and Cancer incidence and mortality worldwide: sources, methods and major patterns in GLOBOCAN 2012. Int J Cancer 2015; 136: E359-386.

[2] Bruix J, Reig M and Sherman M. Evidence-Based Diagnosis, Staging, and Treatment of Patients With Hepatocellular Carcinoma. Gastroenterology 2016; 150: 835-853.

[3] Roayaie S, Jibara G, Tabrizian P, Park JW, Yang J, Yan L, Schwartz M, Han G, Izzo F, Chen M, Blanc JF, Johnson P, Kudo M, Roberts LR and Sherman M. The role of hepatic resection in the treatment of hepatocellular cancer. Hepatology 2015; 62: 440-451.

[4] Zhou L, Huang Y, Li J and Wang Z. The mTOR pathway is associated with the poor prognosis of human hepatocellular carcinoma. Med Oncol 2010; 27: 255-261.

[5] Villanueva A, Hoshida Y, Battiston C, Tovar V, Sia D, Alsinet C, Cornella H, Liberzon A, Kobayashi M, Kumada H, Thung SN, Bruix J, Newell P, April C, Fan JB, Roayaie S, Mazzaferro V, Schwartz ME and Llovet JM. Combining clinical, pathology, and gene expression data to predict recurrence of hepatocellular carcinoma. Gastroenterology 2011; 140: 1501-1512 e1502.

Engels EA. C-reactive protein and risk of lung cancer. J Clin Oncol 2010; 28: 2719-2726.

[7] Elinav E, Nowarski R, Thaiss CA, Hu B, Jin C and Flavell RA. Inflammation-induced cancer: crosstalk between tumours, immune cells and microorganisms. Nat Rev Cancer 2013; 13: 759-771.

[8] Antonioli L, Blandizzi C, Pacher P and Hasko G. Immunity, inflammation and cancer: a leading role for adenosine. Nat Rev Cancer 2013; 13: 842-857. 
276 [9] Ratnam NM, Peterson JM, Talbert EE, Ladner KJ, Rajasekera PV, Schmidt CR, Dillhoff ME, Swanson BJ,

277

278

279

280

281

282

283

284

285

286

287

288

289

290

291

292

293

294

295

296

297

298

299

300

301

302

303

304

305

306

307

308

309

310

311

312

313

314
Haverick E, Kladney RD, Williams TM, Leone GW, Wang DJ and Guttridge DC. NF-kappaB regulates GDF-15 to suppress macrophage surveillance during early tumor development. J Clin Invest 2017; 127: 3796-3809.

[10] Izumi K, Fang LY, Mizokami A, Namiki M, Li L, Lin WJ and Chang C. Targeting the androgen receptor with siRNA promotes prostate cancer metastasis through enhanced macrophage recruitment via CCL2/CCR2-induced STAT3 activation. EMBO Mol Med 2013; 5: 1383-1401.

[11] Balkwill F. Tumour necrosis factor and cancer. Nat Rev Cancer 2009; 9: 361-371.

[12] Manfroi B, McKee T, Mayol JF, Tabruyn S, Moret S, Villiers C, Righini C, Dyer M, Callanan M, Schneider P, Tzankov A, Matthes T, Sturm N and Huard B. CXCL-8/IL8 Produced by Diffuse Large B-cell Lymphomas Recruits Neutrophils Expressing a Proliferation-Inducing Ligand APRIL. Cancer Res 2017; 77: 1097-1107.

[13] He G, Dhar D, Nakagawa H, Font-Burgada J, Ogata H, Jiang Y, Shalapour S, Seki E, Yost SE, Jepsen K, Frazer KA, Harismendy O, Hatziapostolou M, Iliopoulos D, Suetsugu A, Hoffman RM, Tateishi R, Koike $\mathrm{K}$ and Karin M. Identification of liver cancer progenitors whose malignant progression depends on autocrine IL-6 signaling. Cell 2013; 155: 384-396.

[14] Dalpiaz O, Krieger D, Ehrlich GC, Pohlmann K, Stojakovic T, Pummer K, Zigeuner R, Pichler M and Hutterer GC. Validation of the Preoperative Platelet-to-Lymphocyte Ratio as a Prognostic Factor in a European Cohort of Patients with Upper Tract Urothelial Carcinoma. Urol Int 2017; 98: 320-327.

[15] McNamara MG, Templeton AJ, Maganti M, Walter T, Horgan AM, McKeever L, Min T, Amir E and Knox JJ. Neutrophil/lymphocyte ratio as a prognostic factor in biliary tract cancer. Eur J Cancer 2014; 50: 1581-1589.

[16] Tao YM, Huang JL, Zeng S, Zhang S, Fan XG, Wang ZM, Yang HX, Yuan XH, Wang P, Wu F, Luo J, Zeng DY and Shen H. BTB/POZ domain-containing protein 7: epithelial-mesenchymal transition promoter and prognostic biomarker of hepatocellular carcinoma. Hepatology 2013; 57: 2326-2337.

[17] Hu K, Wang ZM, Li JN, Zhang S, Xiao ZF and Tao YM. CLEC1B Expression and PD-L1 Expression Predict Clinical Outcome in Hepatocellular Carcinoma with Tumor Hemorrhage. Transl Oncol 2018; 11: 552-558.

[18] Sanford DE, Belt BA, Panni RZ, Mayer A, Deshpande AD, Carpenter D, Mitchem JB, Plambeck-Suess SM, Worley LA, Goetz BD, Wang-Gillam A, Eberlein TJ, Denardo DG, Goedegebuure SP and Linehan DC. Inflammatory monocyte mobilization decreases patient survival in pancreatic cancer: a role for targeting the CCL2/CCR2 axis. Clin Cancer Res 2013; 19: 3404-3415.

[19] Pribluda A, Elyada E, Wiener Z, Hamza H, Goldstein RE, Biton M, Burstain I, Morgenstern Y, Brachya G, Billauer H, Biton S, Snir-Alkalay I, Vucic D, Schlereth K, Mernberger M, Stiewe T, Oren M, Alitalo K, Pikarsky E and Ben-Neriah Y. A senescence-inflammatory switch from cancer-inhibitory to cancerpromoting mechanism. Cancer Cell 2013; 24: 242-256.

[20] Lu SD, Wang YY, Peng NF, Peng YC, Zhong JH, Qin HG, Xiang BD, You XM, Ma L and Li LQ. Preoperative Ratio of Neutrophils to Lymphocytes Predicts Postresection Survival in Selected Patients With Early or Intermediate Stage Hepatocellular Carcinoma. Medicine (Baltimore) 2016; 95: e2722. 
315 [21] Feng JF, Huang Y and Chen QX. Preoperative platelet lymphocyte ratio (PLR) is superior to neutrophil

316

317

318

319

320

321

322

323

324

325

326

327

328

329

330

331

332

333

334

335 Figure legends:

336

337

338

339

340

341

342

343

344

345

346

347 Figure 4. Analysis of the relationship between NLR, PLR and tumor size. PLR and CRP in surgical renal cell carcinoma patients. World J Urol 2017; 35: 261-270.

[23] Sanchez-Salcedo P, de-Torres JP, Martinez-Urbistondo D, Gonzalez-Gutierrez J, Berto J, Campo A, for lung cancer development. Lung Cancer 2016; 97: 28-34. 128. Blockade. Cell 2017; 170: 1120-1133 e1117. of NLR was 0.63 . B. The ROC area of PLR was 0.67. C The ROC area of LNR was 0.53 . RFS and OS (A and C); patients with PLR >128.1 have a shorter RFS and OS (B and D).

Figure 3. Effect of combined NLR and PLR on RFS and OS in patients with HCC who underwent hepatectomy. The NLR-high and PLR-high group had the worst RFS (median 12 (median 14.5 months) and OS (median 23 months).

[22] Hu H, Yao X, Xie X, Wu X, Zheng C, Xia W and Ma S. Prognostic value of preoperative NLR, dNLR, Alcaide AB and Zulueta JJ. The neutrophil to lymphocyte and platelet to lymphocyte ratios as biomarkers

[24] Rizvi NA, Hellmann MD, Snyder A, Kvistborg P, Makarov V, Havel JJ, Lee W, Yuan J, Wong P, Ho TS, Miller ML, Rekhtman N, Moreira AL, Ibrahim F, Bruggeman C, Gasmi B, Zappasodi R, Maeda Y, Sander C, Garon EB, Merghoub T, Wolchok JD, Schumacher TN and Chan TA. Cancer immunology. Mutational landscape determines sensitivity to PD-1 blockade in non-small cell lung cancer. Science 2015; 348: $124-$

[25] Wei SC, Levine JH, Cogdill AP, Zhao Y, Anang NAS, Andrews MC, Sharma P, Wang J, Wargo JA, Pe'er D and Allison JP. Distinct Cellular Mechanisms Underlie Anti-CTLA-4 and Anti-PD-1 Checkpoint

Figure 1. The ROC curves of the NLR, PLR, and LMR in patients with HCC. A. The ROC area

Figure 2. Kaplan-Meier survival analysis indicates that patients with NLR $>2.92$ have a shorter months) and OS (median 18 months), and the NLR-low and PLR-low group had the best RFS 
348 We divided the tumors into three groups according to size: $\leq 3 \mathrm{~cm}$ group, between $3-10 \mathrm{~cm}$

349 group, and $\geq 10 \mathrm{~cm}$ group. A: The mean NLRs in the tumor $\leq 3 \mathrm{~cm}$ group, $3-10 \mathrm{~cm}$ group and $\geq 10$

$350 \mathrm{~cm}$ group were $(2.32 \pm 0.15),(3.23 \pm 0.17)$, and $(4.03 \pm 0.38)$, respectively. B: the mean PLRs

351 were $(90.21 \pm 6.44),(128.5 \pm 5.4)$, and $(157 \pm 13.41)$, respectively. ${ }^{* *}$ means $P<0.05$.

352

353 Figure 5. Analysis of the relationship between NLR, PLR and BCLC stage. A: The mean NLR

354 values of the BCLC $0, A, B$, and $\mathrm{C}$ stages were $(1.70 \pm 0.14),(2.93 \pm 0.13),(3.05 \pm 0.26)$, and

355 (4.82 \pm 0.65$)$, respectively. B: The mean PLR values of the BCLC $0, A, B$, and C stages were

356 (81.93 \pm 10.68$),(122.1 \pm 5.61),(122.5 \pm 8.91)$, and (149.2 \pm 16.13$)$, respectively. ${ }^{* *}$ means $P<$ 3570.05 . 
Figure 1

The ROC curves of the NLR, PLR, and LMR in patients with HCC.

The ROC curves of the NLR, PLR, and LMR in patients with HCC. A. The ROC area of NLR was 0.63. B. The ROC area of PLR was 0.67. C The ROC area of LNR was 0.53 .

A

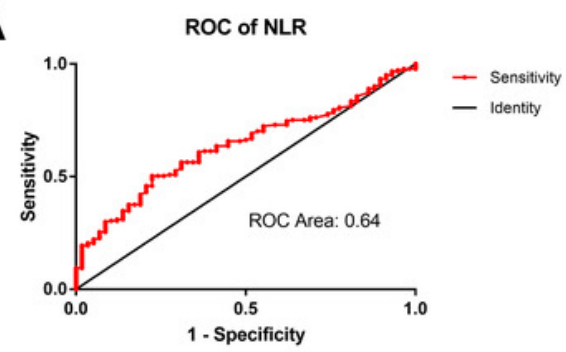

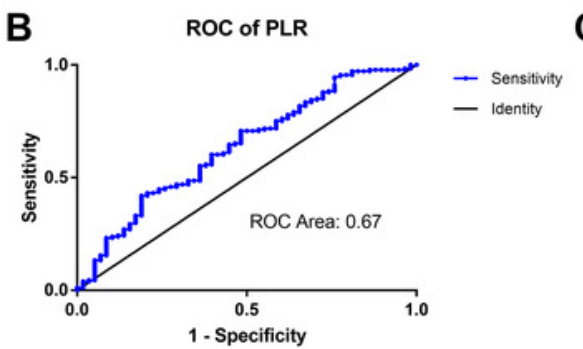

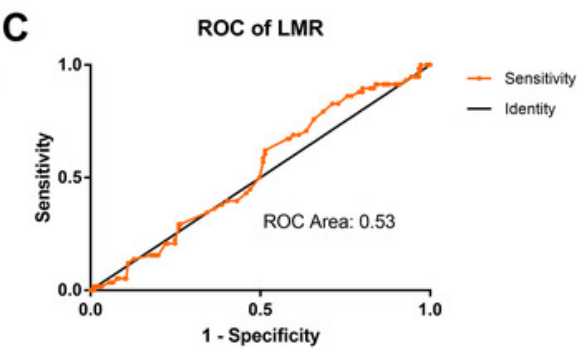


Figure 2

Kaplan-Meiersurvival analysis indicates that patients with NLR $>2.92$ have a shorter RFSand OS (A and C); patients with PLR>128.1 have a shorter RFS and OS (B andD).

Kaplan-Meier survival analysis indicates that patients with NLR $>2.92$ have a shorter RFS and OS (A and C); patients with PLR $>128.1$ have a shorter RFS and OS (B and D).
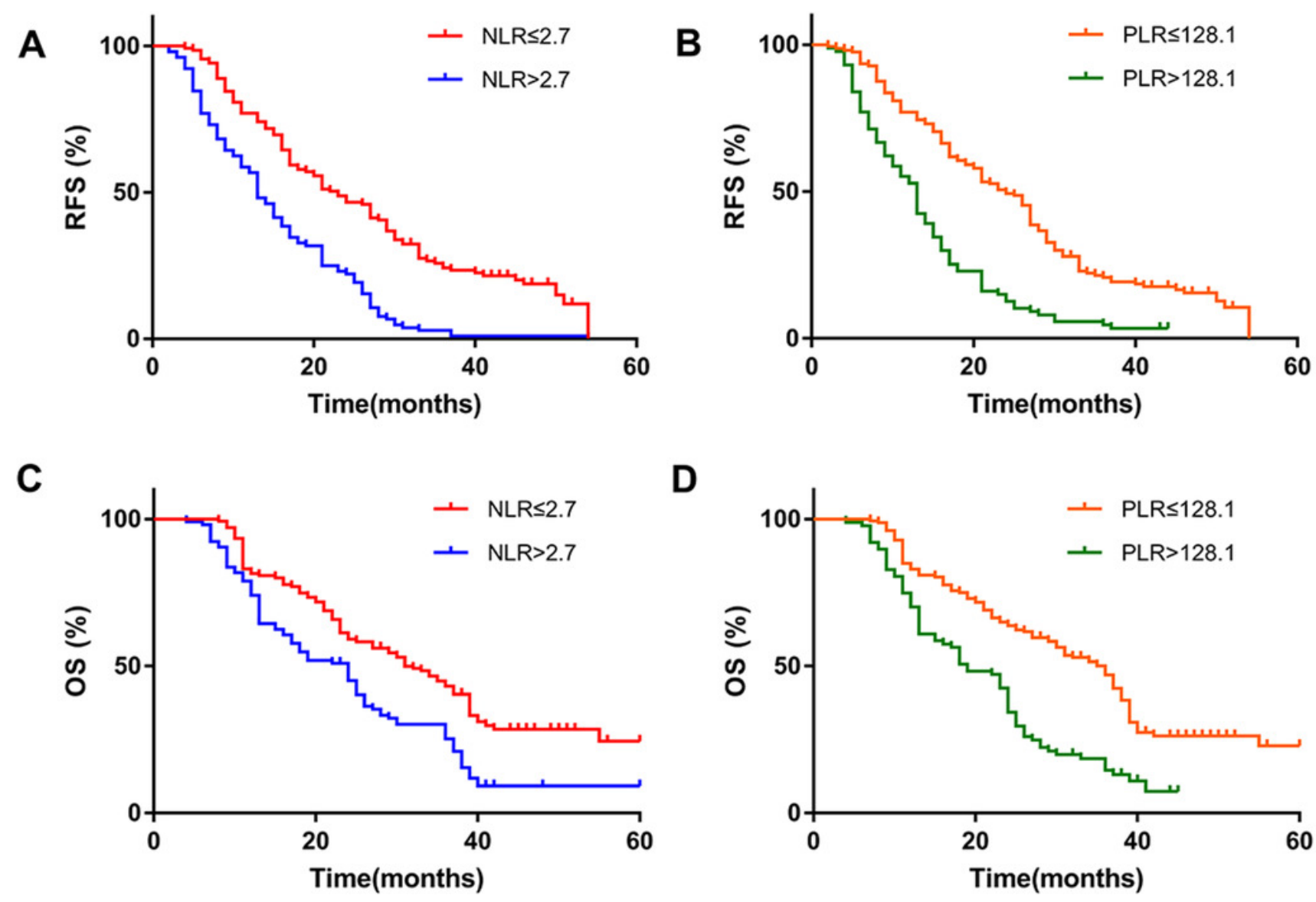
Figure 3

Effect of combined NLR and PLR on RFS and OS in HCC patients who underwent hepatectomy.

The NLR-high and PLR-high group had the worst RFS (median 12 months) and OS (median 18 months), and the NLR-low and PLR-low group had the best RFS (median 14.5 months) and OS (median 23 months).
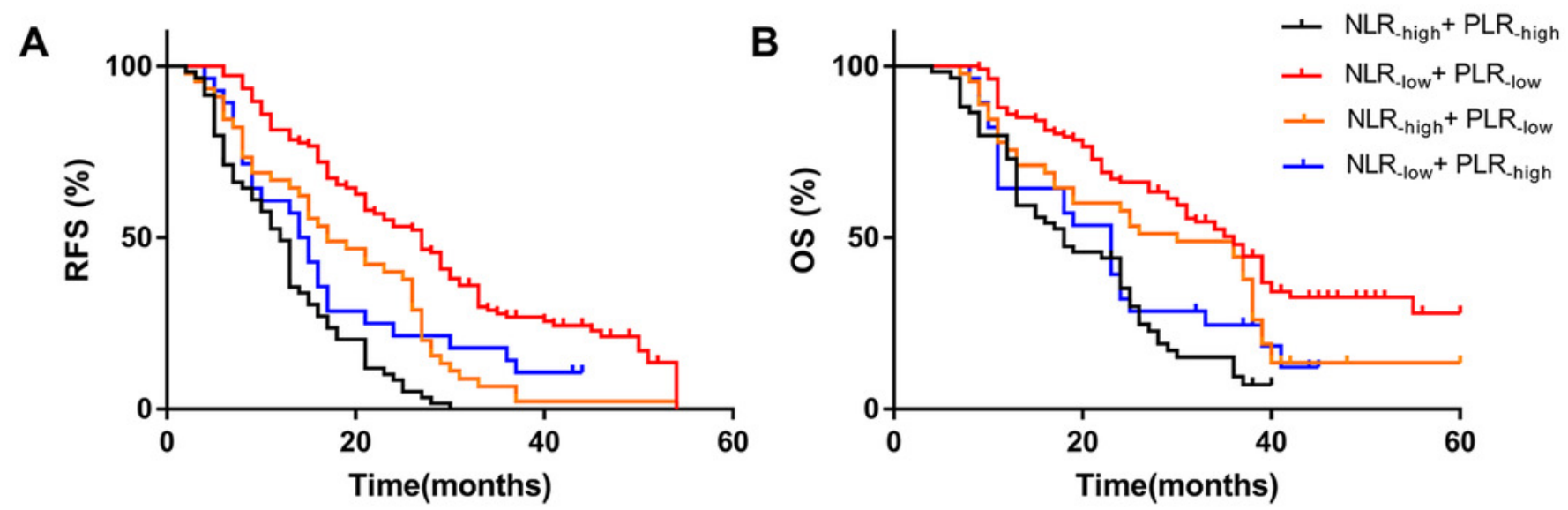
Figure 4

Analysis of the relationship between NLR, PLR and tumor size.

We divided the tumors into three groups according to size: $\leq 3 \mathrm{~cm}$ group, between $3-10 \mathrm{~cm}$ group, and $\geq 10$ $\mathrm{cm}$ group. A: The mean NLRs in the tumor $\leq 3 \mathrm{~cm}$ group, $3-10 \mathrm{~cm}$ group and $\geq 10 \mathrm{~cm}$ group were $(2.32 \pm$ $0.15),(3.23 \pm 0.17)$, and $(4.03 \pm 0.38)$, respectively. B: the mean PLRs were $(90.21 \pm 6.44),(128.5 \pm 5.4)$, and $(157 \pm 13.41)$, respectively. ${ }^{* *}$ means $P \square 0.05$.

A

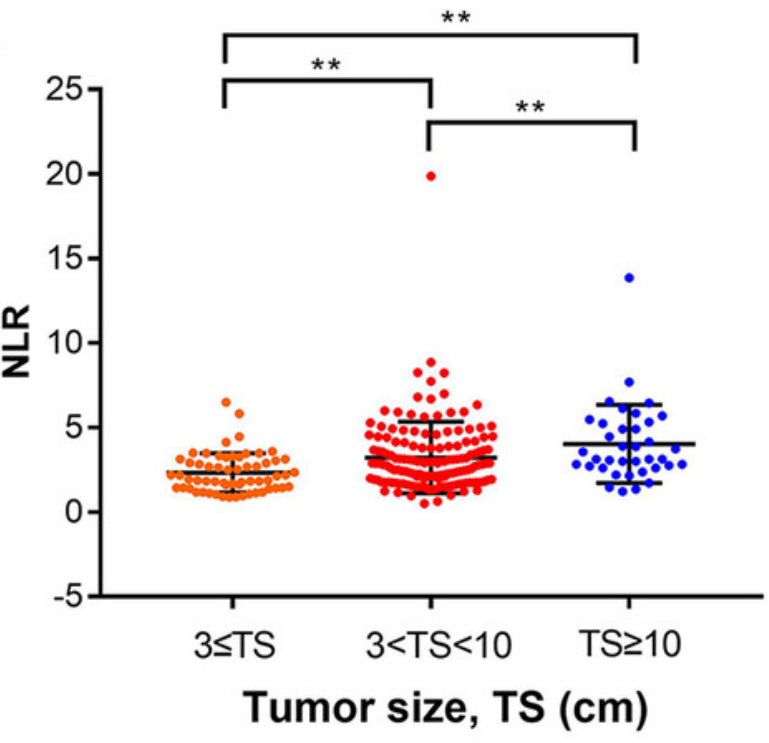

B

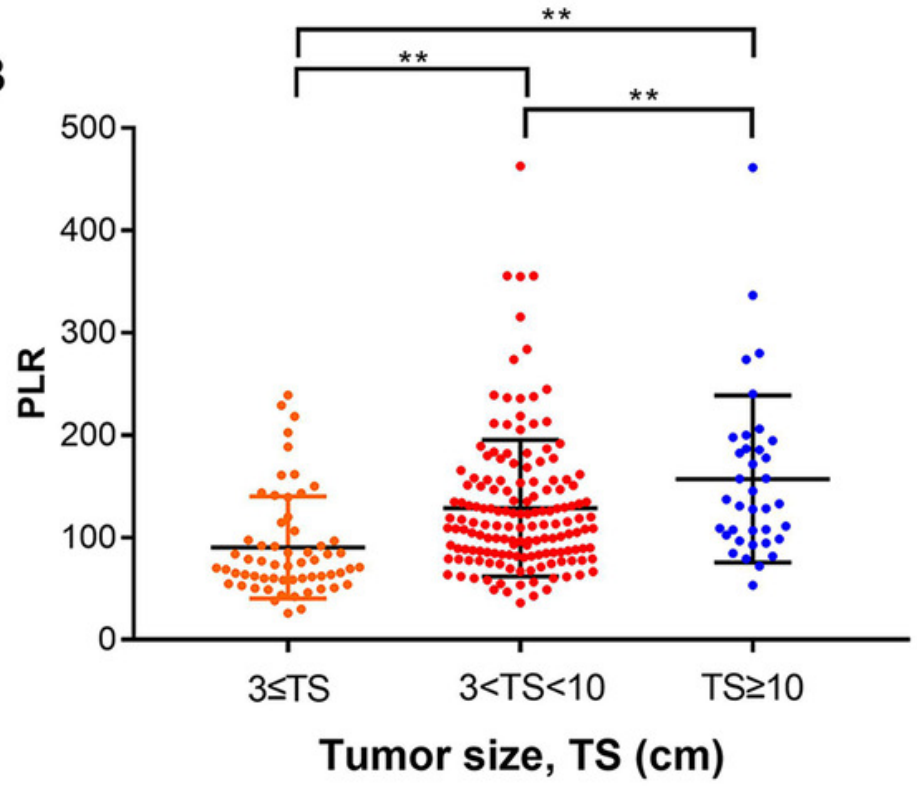


Figure 5

Analysis of the relationship between NLR, PLR and BCLC stage.

Analysis of the relationship between NLR, PLR and BCLC stage. A : The mean NLR values of the BCLC $0, A, B$, and C stages were (1.70 \pm 0.14$),(2.93 \pm 0.13),(3.05 \pm 0.26)$, and $(4.82 \pm$ $0.65)$, respectively. $B$ : The mean PLR values of the $B C L C D, A, B$, and $C$ stages were $(81.93 \pm$ 10.68), $(122.1 \pm 5.61),(122.5 \pm 8.91)$, and $(149.2 \pm 16.13)$, respectively. ${ }^{* *}$ means $P \square 0.05$.
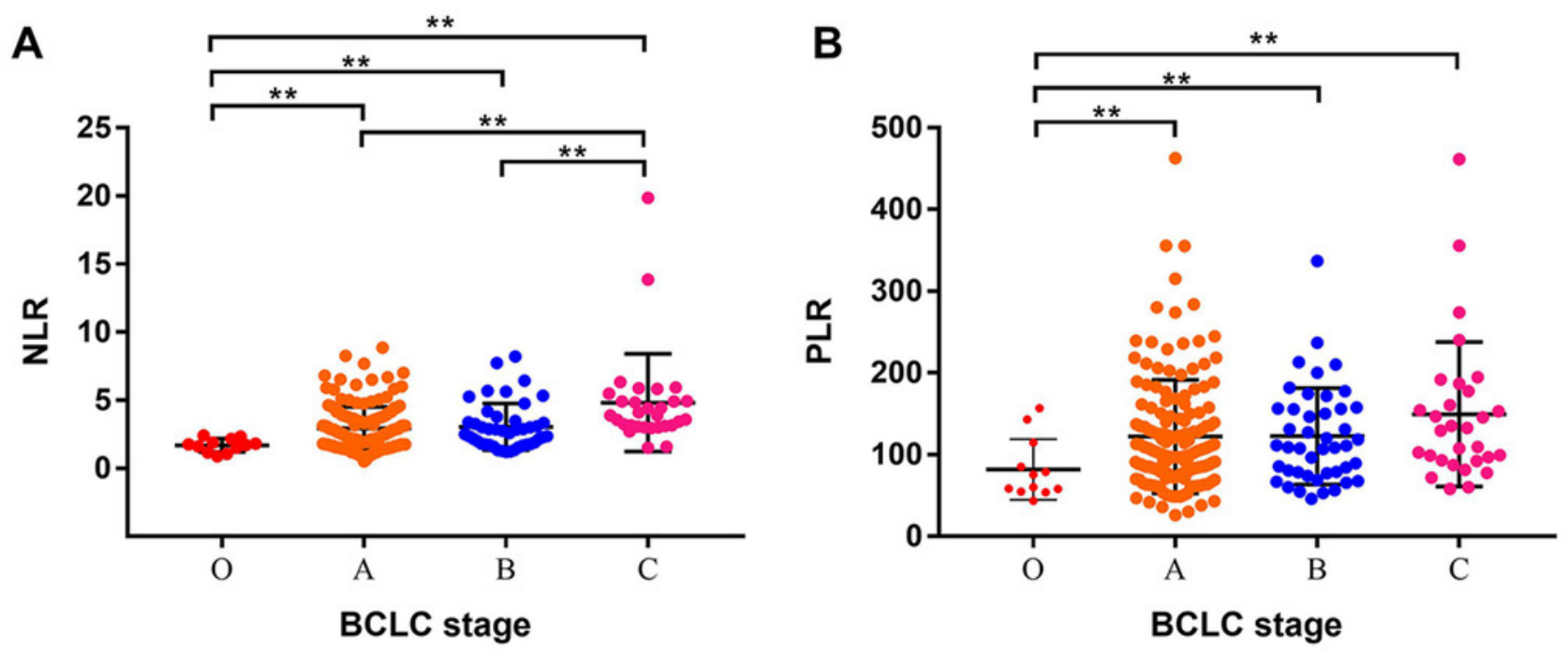


\section{Table $\mathbf{1}$ (on next page)}

HCC patients $(n=239)$ categorized by NLR, PLR and their clinical pathologic characteristics. 
1 Table 1. HCC Patients $(n=239)$ Categorized by NLR, PLR and Their Clinical Pathologic

\begin{tabular}{|c|c|c|c|c|c|c|c|}
\hline \multirow{2}{*}{ Clinical character } & & \multicolumn{3}{|c|}{ NLR } & \multicolumn{3}{|c|}{ PLR } \\
\hline & & $\leq 2.92(\mathrm{n}=135)$ & $>2.92(n=104)$ & $P$-value & $\leq 128.1(n=152)$ & $>128(n=87)$ & $P$-value \\
\hline Age, years & & $49.0 \pm 12.47$ & $51.28 \pm 11.33$ & 0.34 & $48.82 \pm 11.21$ & $52.54 \pm 13.01$ & 0.02 \\
\hline Serum albumin, $g / \mathrm{L}$ & & $41.36 \pm 0.38$ & $41.49 \pm 0.5$ & 0.59 & $41.82 \pm 0.39$ & $40.97 \pm 0.49$ & 0.18 \\
\hline Tumor size, $\mathrm{cm}$ & & $5.01 \pm 0.26$ & $6.99 \pm 0.36$ & 0.00 & $5.18 \pm 0.25$ & $7.17 \pm 0.39$ & 0.00 \\
\hline Platelet, $10^{\wedge} 9 / \mathrm{L}$ & & $156.0 \pm 6.92$ & $167.7 \pm 6.89$ & 0.25 & $133.4 \pm 4.43$ & $209.7 \pm 9.03$ & 0.00 \\
\hline $\mathrm{TBil}, \mu \mathrm{mol} / \mathrm{L}$ & & $14.2 \pm 0.63$ & $16.24 \pm 1.52$ & 0.18 & $14.56 \pm 0.57$ & $16.02 \pm 1.82$ & 0.35 \\
\hline $\mathrm{ALT}, \mathrm{U} / \mathrm{L}$ & & $41.06 \pm 2.77$ & $44.91 \pm 3.19$ & 0.36 & $41.66 \pm 2.39$ & $44.62 \pm 3.95$ & 0.49 \\
\hline $\mathrm{AST}, \mathrm{U} / \mathrm{L}$ & & $44.51 \pm 2.72$ & $50.18 \pm 3.05$ & 0.17 & $44.18 \pm 2.14$ & $52.28 \pm 4.12$ & 0.06 \\
\hline $\mathrm{PT}, \mathrm{s}$ & & $13.22 \pm 0.10$ & $13.33 \pm 0.10$ & 0.57 & $13.38 \pm 0.09$ & $13.06 \pm 0.09$ & 0.02 \\
\hline Gender & Male & 115 & 85 & 0.49 & 132 & 68 & 0.10 \\
\hline$\square$ & Female & 20 & 19 & & 20 & 19 & \\
\hline \multirow[t]{2}{*}{ HBsAg } & Negative & 23 & 14 & 0.48 & 19 & 18 & 0.10 \\
\hline & Positive & 112 & 90 & & 133 & 69 & \\
\hline AFP, ng/mL & $\leq 20$ & 50 & 34 & 0.49 & 45 & 39 & 0.02 \\
\hline$\square$ & $>20$ & 85 & 70 & & 107 & 48 & \\
\hline Liver cirrhosis & No & 33 & 32 & 0.31 & 41 & 24 & 0.92 \\
\hline$\square$ & Yes & 102 & 72 & & 111 & 63 & \\
\hline Tumor encapsulation & No & 96 & 72 & 0.78 & 110 & 58 & 0.37 \\
\hline$\square$ & Yes & 39 & 32 & & 42 & 29 & \\
\hline Tumor number & Single & 105 & 77 & 0.54 & 119 & 63 & 0.35 \\
\hline$\square$ & Multiple & 30 & 27 & & 33 & 24 & \\
\hline Satellite nodules & No & 125 & 96 & 0.98 & 146 & 75 & 0.01 \\
\hline$\square$ & Yes & 10 & 8 & & 6 & 12 & \\
\hline Edmondson grade & $\mathrm{I}-\mathrm{II}$ & 103 & 83 & 0.53 & 116 & 70 & 0.52 \\
\hline$\square$ & III-IV & 32 & 21 & & 36 & 17 & \\
\hline \multirow[t]{2}{*}{ BCLC stage } & 0 & 11 & 0 & 0.00 & 10 & 1 & 0.04 \\
\hline & A & 93 & 60 & & 101 & 52 & \\
\hline$\square$ & $\mathrm{B}$ & 28 & 17 & & 27 & 18 & \\
\hline$\square$ & $\mathrm{C}$ & 3 & 27 & & 14 & 16 & \\
\hline TNM stage & I & 95 & 48 & 0.00 & 102 & 41 & 0.01 \\
\hline$\square$ & II & 30 & 19 & & 27 & 22 & \\
\hline$\square$ & III & 10 & 37 & & 23 & 24 & \\
\hline
\end{tabular}

2 Characteristics.

3 NLR, neutrophil-to-lymphocyte ratio; HBsAg, hepatitis B surface antigen; AFP, $\alpha$-fetoprotein; TNM, tumor-node-

4 metastasis; TBil, total bilirubin; PT, Prothrombin time; CTP, Child-Turcotte-Pugh; BCLC stage: The Barcelona Clinic 
5 Liver Cancer staging; ALT, glutamic-pyruvic transaminase; AST, glutamic oxalacetic transaminase. 


\section{Table 2 (on next page)}

Univariate and multivariate analyses of prognostic factors with RFS and OS in patients with HCC ( $n=239)$. 
1 Table 2. Univariate and multivariate analyses of prognostic factors with RFS and OS in patients with HCC (n $2=239$ ).

\begin{tabular}{|c|c|c|c|c|}
\hline \multirow{2}{*}{ Clinicopathologic variable } & RFS & $\square$ & OS & $\square$ \\
\hline & HR $(95 \% \mathrm{CI})$ & $P$-value & HR $(95 \%$ CI $)$ & $P$-value \\
\hline \multicolumn{5}{|l|}{ Univariate analysis } \\
\hline Gender (male vs. female) & $2.40(0.54-10.64)$ & 0.25 & $1.93(0.77-4.89)$ & 0.16 \\
\hline Age, years ( $>60$ vs. $\leq 60)$ & $1.00(0.97-1.04)$ & 0.86 & $0.98(0.96-1.00)$ & 0.21 \\
\hline Serum albumin, g/L ( $\leq 35$ vs. $>35$ ) & $1.00(0.88-1.04)$ & 0.28 & $0.99(0.93-1.05)$ & 0.73 \\
\hline Platelet,10^9/L ( $\leq 160$ vs. $>160)$ & $1.00(0.99-1.008)$ & 0.50 & $1.01(1.001-1.01)$ & 0.02 \\
\hline TBil, $\mu \mathrm{mol} / \mathrm{L}(\leq 17.1$ vs. $>17.1$ ) & $1.02(0.97-1.05)$ & 0.83 & $1.01(0.98-1.05)$ & 0.52 \\
\hline ALT, U/L ( $\leq 50$ vs. $>50)$ & $1.01(0.99-103)$ & 0.40 & $1.01(0.98-1.03)$ & 0.09 \\
\hline AST, U/L ( $\leq 40$ vs. $>40)$ & $1.01(0.99-1.03)$ & 0.29 & $1.02(1.00-1.03)$ & 0.05 \\
\hline PT, s ( $\leq 13.2$ vs. $>13.2)$ & $0.89(0.60-1.31)$ & 0.54 & $0.82(0.61-1.08)$ & 0.16 \\
\hline AFP, ng/mL (>20 vs. $\leq 20)$ & $2.18(0.95-5.03)$ & 0.07 & $1.72(0.94-3.15)$ & 0.08 \\
\hline HBV (presence vs. absence) & $4.86(0.64-37.04)$ & 0.13 & $1.19(0.54-2.63)$ & 0.67 \\
\hline NLR (>2.92 vs. $\leq 2.92)$ & $2.85(1.63-4.93)$ & $<0.01$ & $1.48(1.16-1.88)$ & $<0.01$ \\
\hline PLR (>128.1 vs. $\leq 128.1)$ & $1.01(1.00-1.02)$ & 0.012 & $1.01(1.00-1.013)$ & 0.014 \\
\hline BCLC stage (C vs. 0/A/B) & $3.01(1.39-6.50)$ & $<0.01$ & $2.43(0.98-5.98)$ & $<0.01$ \\
\hline TNM stage (II/III vs. I) & $6.57(0.87-49.8)$ & 0.01 & $19.42(2.61-144.3)$ & $<0.01$ \\
\hline Tumor number (multiple vs. single) & $2.48(0.71-8.56)$ & 0.15 & $2.78(1.18-6.54)$ & 0.02 \\
\hline Edmondson grade (III/IV vs. I/II) & $1.56(0.51-4.76)$ & 0.44 & $1.12(0.54-2.32)$ & 0.75 \\
\hline Tumor size, cm ( $>5$ vs. $\leq 5)$ & $1.30(1.08-1.57)$ & $<0.01$ & $1.42(1.23-1.63)$ & $<0.01$ \\
\hline Satellite nodules (presence vs. absence) & $4.27(2.55-7.14)$ & $<0.01$ & $4.42(2.66-7.33)$ & $<0.01$ \\
\hline Tumor encapsulation (none vs. complete) & $1.34(0.53-3.62)$ & 0.51 & $1.45(0.73-2.85)$ & 0.29 \\
\hline Liver cirrhosis (presence vs. absence) & $1.30(0.53-3.17)$ & 0.57 & $1.03(0.53-1.99)$ & 0.94 \\
\hline Hospital stay, d & $1.02(0.88-1.19)$ & 0.75 & $1.04(0.94-1.16)$ & 0.45 \\
\hline \multicolumn{5}{|l|}{ Multivariate analysis } \\
\hline Platelet, $10^{\wedge} 9 / \mathrm{L}(\leq 160$ vs. $>160)$ & NA & & $0.99(0.99-1.00)$ & 0.46 \\
\hline AST, U/L ( $\leq 40$ vs. $>40)$ & NA & & $1.00(0.99-1.01)$ & 0.42 \\
\hline AFP, ng/mL (>20 vs. $\leq 20)$ & $1.39(1.05-1.88)$ & 0.03 & $1.37(1.01-1.86)$ & 0.04 \\
\hline Tumor size, cm (>5 vs. $\leq 5)$ & $1.10(1.05-1.15)$ & 0.01 & $1.10(1.05-1.16)$ & 0.01 \\
\hline NLR (>2.92 vs. $\leq 2.92)$ & $1.16(1.06-1.26)$ & $<0.01$ & $1.14(1.04-1.25)$ & $<0.01$ \\
\hline $\operatorname{PLR}(>128.1$ vs. $\leq 128.1)$ & $1.01(1.00-1.01)$ & $<0.01$ & $1.00(1.00-1.01)$ & $<0.01$ \\
\hline TNM (II/III vs. I) & $1.40(0.77-2.53)$ & 0.27 & $1.39(0.76-2.55)$ & 0.28 \\
\hline Tumor number (multiple vs. single) & $1.34(0.92-1.96)$ & 0.13 & $1.33(0.91-1.95)$ & 0.15 \\
\hline Satellite nodules (presence vs. absence) & $3.03(1.62-5.65)$ & 0.00 & $2.98(1.59-5.57)$ & 0.00 \\
\hline
\end{tabular}


3 NLR, neutrophil-to-lymphocyte ratio; HBsAg, hepatitis B surface antigen; AFP, $\alpha$-fetoprotein; TNM, tumor-node4 metastasis; TBil, total bilirubin; PT, Prothrombin time; CTP, Child-Turcotte-Pugh; BCLC stage: The Barcelona

5 Clinic Liver Cancer staging; ALT, glutamic-pyruvic transaminase; AST, glutamic oxalacetic transaminase. 